\title{
A manual reduction of hernia under analgesia/sedation (Taxis) in the acute inguinal hernia: a useful technique in COVID-19 times to reduce the need for emergency surgery-a literature review
}

\author{
B. East ${ }^{1,2}$ (D) M. Pawlak ${ }^{3} \cdot$ A. C. de Beaux ${ }^{4}$
}

Received: 28 April 2020 / Accepted: 25 May 2020 / Published online: 29 May 2020

○) Springer-Verlag France SAS, part of Springer Nature 2020

\begin{abstract}
Background Acute IH is a common surgical presentation. Despite new guidelines being published recently, a number of important questions remained unanswered including the role of taxis, as initial non-operative management. This is particularly relevant now due to the possibility of a lack of immediate surgical care as a result of COVID-19. The aim of this review is to assess the role of taxis in the management of emergency inguinal hernias.

Methods A review of the literature was undertaken. Available literature published until March 2019 was obtained and reviewed. 32,021 papers were identified, only 9 were of sufficient value to be used.

Results There was a large discrepancy in the terminology of incarcerated/strangulated used. Taxis can be safely attempted early after the onset of symptoms and is effective in about $70 \%$ of patients. The possibility of reduction en-mass should be kept in mind. Definitive surgery to repair the hernia can be delayed by weeks until such time as surgery can be safely arranged. Conclusions The use of taxis in emergency inguinal hernia is a useful first line of treatment in areas or situations where surgical care is not immediately available, including the COVID-19 pandemic. Emergency surgery remains the mainstay of management in the strangulated hernia setting.
\end{abstract}

Keywords Inguinal hernia $\cdot$ Incarcerated $\cdot$ Strangulated $\cdot$ Surgery

\section{Introduction}

Patients presenting with an acute inguinal hernia (IH) remain a common surgical emergency. The popular "watch and wait" policy for IH is increasing the emergency presentation

B. East

barbora.east@gmail.com

M. Pawlak

pawlakmd@gmail.com

A. C. de Beaux

adebeaux@doctors.org.uk

1 3rd Department of Surgery, 1st Faculty of Medicine, Charles University, Motol University Hospital, V uvalu 84, Prague 5, 15006 Prague, Czech Republic

2 2nd Faculty of Medicine, Charles University, Prague, Czech Republic

3 Northern Devon Healthcare NHS Trust, Barnstaple, UK

4 Department of General Surgery, Royal Infirmary of Edinburgh, Edinburgh, UK of such hernias [1]. The management of such patients is associated with significant morbidity and indeed mortality. The recently published HerniaSurge guidelines [2] on groin hernias included a short chapter on the emergency groin hernia. However, a number of important questions relating to the management of such patients remain, and the role of taxis (manual reduction of a hernia under analgesia/sedation) was not mentioned. Furthermore, the WSES guidelines for emergency repair of complicated abdominal wall hernias and their recent update does not mention taxis either [14].

In the acute painful $\mathrm{IH}$, as long as it is reducible, there is no surgical emergency. However, if the hernia is painful and irreducible on simple clinical examination, the clinical concern should be of possible strangulation of the hernia contents. Reduction of the hernia contents with analgesia/ sedation in the emergency setting may be an option worth considering. The place of formal taxis is not well defined. This is particularly true in the elderly, with their associated comorbidities, and often antiplatelet or anticoagulant therapy, that can lead to delays or might be a contraindication to proceeding directly to surgery. And indeed, concerns about 
surgery during pandemics such as the ongoing COVID-19, make the role of taxis a useful adjunct in the care of acute inguinal hernia [3]. At times, hospitals and health services have been overwhelmed, in addition to the individual risk to the patient positive for COVID-19 from general anesthesia. The European Hernia Society have produced a useful guidance paper on this subject [4].

The aim of this study was to perform a review of the literature on the management of IHs presenting as an emergency in adult patients, with a particular focus on the role of taxis.

\section{Methods}

In March 2019 we searched Medline, Scopus, Ovid and Embase databases for papers related to emergency inguinal hernias. Search terms "groin", "inguinal", "strangulated", "incarcerated", "irreducible", "emergency", "groin/inguinal hernia", "taxis" and "reduction" were used. Two of the authors of this study carried out the first search, which resulted in 32,021 hits. We checked both titles and abstracts, with duplicates, case reports, and non-English articles being excluded. We identified 90 publications and obtained them in full-text. Two authors then reviewed the 90 papers and assessed their quality based on the GRADE approach [5] with the help of the third author where no consensus could be reached, 9 papers were included in this review (Fig. 1).

We focused the literature review on data relating to emergency IH incidence, patient pathways, use of taxis, and the associated clinical outcomes. The definitions of clinical outcomes were taken from the papers reviewed, accepting that variation in such definitions are likely to exist between publications.

We have used the HerniaSurge guidelines [2] definition of incarcerated and strangulated hernia. "Incarceration: Inability to reduce the hernia mass into the abdomen and Strangulation: The blood supply to the herniated tissues is compromised".

The aim had been to perform a systematic review of the literature. However, the quality of the data available was such that no statistical analysis was possible.

\section{Results}

This review summarises the 9 papers identified in the literature search.

\section{Incidence of incarceration/strangulation}

The incidence of IH incarceration is difficult to assess. The terms 'incarcerated' and 'strangulated' were used interchangeably in many studies which made the separation of these distinct entities difficult in the analysis. Inguinal hernias account for around 68-95\% (45-85\% indirect, 2-9\% direct, $13-14 \%$ recurrent) of all incarcerated groin hernias $[6,7]$ representing around $1 \%$ of all inguinal hernias undergoing surgical repair [8]. Due to inaccurate use of definitions
Fig. 1 PRISMA flow diagram of the literature search

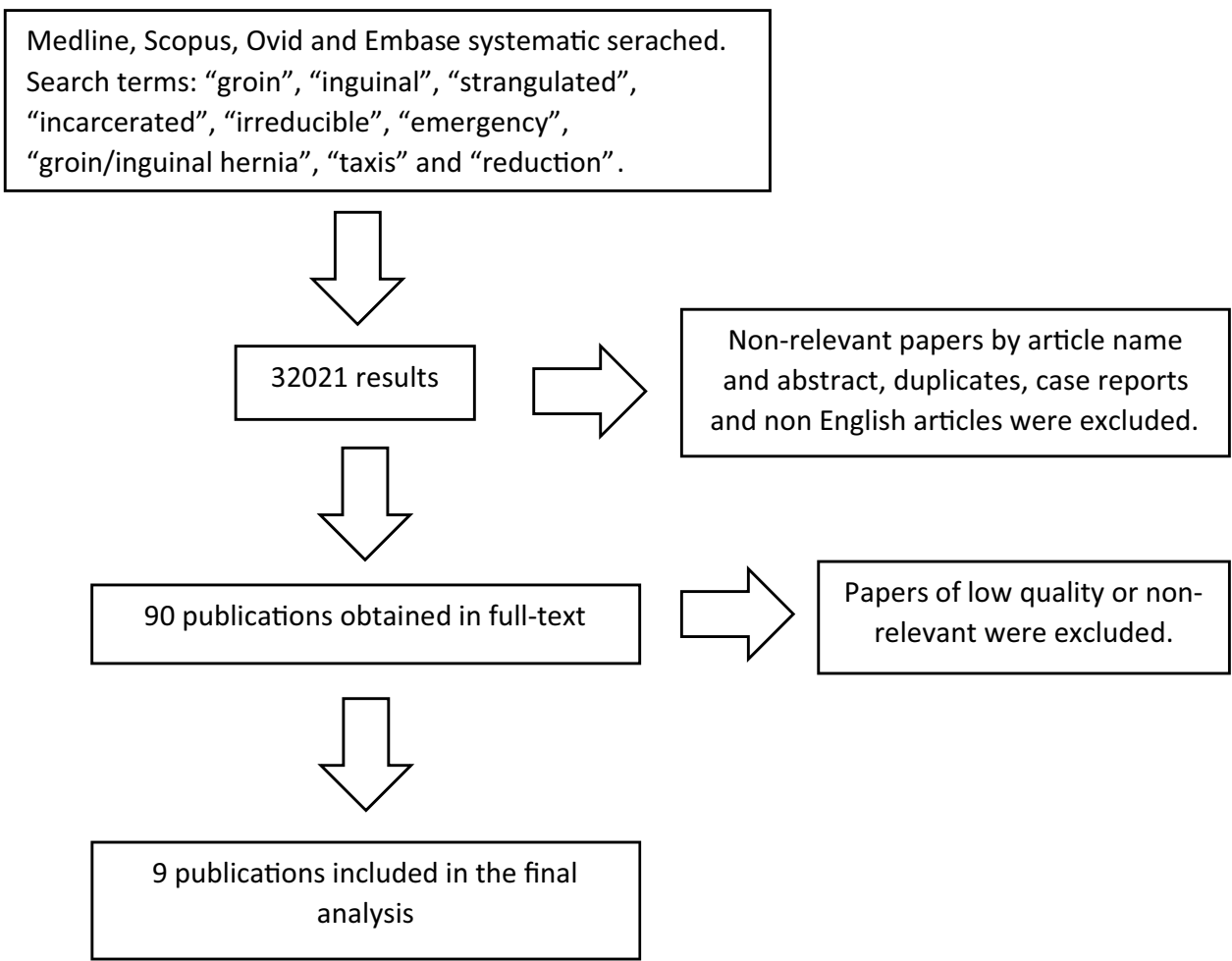


the results reported are very variable. Approximately $10 \%$ of incarcerated indirect and $20 \%$ of direct inguinal hernias require a bowel resection [9]. In one study of suspected strangulated hernias, $10 \%$ contained omentum as the only sac content, $75 \%$ contained small bowel, and $15 \%$ contained colon. Yet only $17.5 \%$ of these hernias actually contained non-viable tissue during surgery of which $75 \%$ were small bowel and 25\% were omentum [7] (Table 1).

\section{Effectiveness of taxis}

Taxis can be successful in up to $70 \%$ of patients presenting with a symptomatic incarcerated IH according to one study [10]. The main factor associated with less chance of successful reduction was the time from the onset of worsening pain in the groin. In all studies identified, hernias containing infarcted bowel could not be reduced by taxis [6]. Reports of reduction-en-masse were rare and were just case resports [11]. If reduction was successful, hernia repair was undertaken during the same admission or within 1 month (Table 1). There appears to be a linear relationship between the time from the onset of symptoms, to strangulation [12]. The likelihood of strangulation and the necessity of bowel resection increases with the time since the onset of symptoms. The risk of strangulation doubles for every $24 \mathrm{~h}$ of delay.

\section{Discussion}

This study identified the relative lack of published evidence on which to base a recommendation of the place of taxis in the management algorithm for acute inguinal hernia. We suggest to attempt taxis (reduction of the hernia) in the acute setting. Two attempts at taxis in the same patient are reported [13], but in general, we suggest attempting this once. If successful, surgery during the same admission or later depending on the local surgical circumstances is acceptable. Clinical contraindications for taxis and therefore indications for emergency surgery include clinical features associated with strangulated hernia content. Bowel obstruction in the absence of these findings is not a contra-indication to perform taxis. Ultrasound-guided hernia reduction has been described as successful in cases where an attempt of taxis had failed. According to one prospective study the rate of emergency operations was reduced from nearly 10 to $2 \%$ due to the higher success rate of taxis under ultrasound guidance [13].

It has been a tradition to keep patients in the hospital for 12-24 $\mathrm{h}$ following taxis to ensure that the patient remained well. One study [10], reported on 31 patients, following successful taxis, discharged at $24 \mathrm{~h}$ and underwent elective surgery on average 4 weeks later. Only one patient was readmitted as an emergency in the interim and discharged after another successful taxis. We suggest that in patients positive with COVID-19, but otherwise well, or at times in all patients when the emergency services are under strain, monitoring for several hours to ensure the analgesia/sedation has worn off. Then prompt discharge from hospital with advice on symptoms/signs to look out for in the next $24 \mathrm{~h}$ (associated with bowel ischemia/obstruction) that should prompt return to the hospital if they occur. [4]

\section{Limitations}

This review is a summary of the available literature on the role of taxis in a patient with incarcerated/strangulated inguinal hernia. Other types of hernias were excluded from this review. Where possible, we have extracted only data regarding inguinal hernias from studies reporting on other hernia types such as femoral, incisional, or primary ventral hernias. We have excluded most case reports and studies with insufficient descriptions of methodology, unclear results, and incomplete data. However several case reports are mentioned in the reference list as for example, with regard to en-masse reduction, we were only able to find case reports. The lack of clear definitions of 'incarcerated' versus 'strangulated' in many reports made the pooling of data from trials impossible.

Implications for future research. We believe that further studies with more accurate use of terminology are needed to give a clearer picture of the true incidence of incarcerated and strangulated hernias. The need for an international registry is clear to inform on the role and importance of taxis as well as reporting on the various complications and long-term results in this condition to aid future management decisions.

\section{Conclusions}

The emergency inguinal hernia remains a common clinical scenario with the time to treatment important, to either reduce the hernia content and prevent progression to strangulation, or to proceed to surgery, if the successful reduction is not possible, or strangulation is already established. Taxis is a safe option to delay surgery in most patients, but the time since onset of symptoms to manual reduction is crucial for prognosis. Minimizing the risks to patients and healthcare workers in the COVID-19 pandemic, make taxis and prompt hospital discharge a very useful adjunct to the care of such patients. 


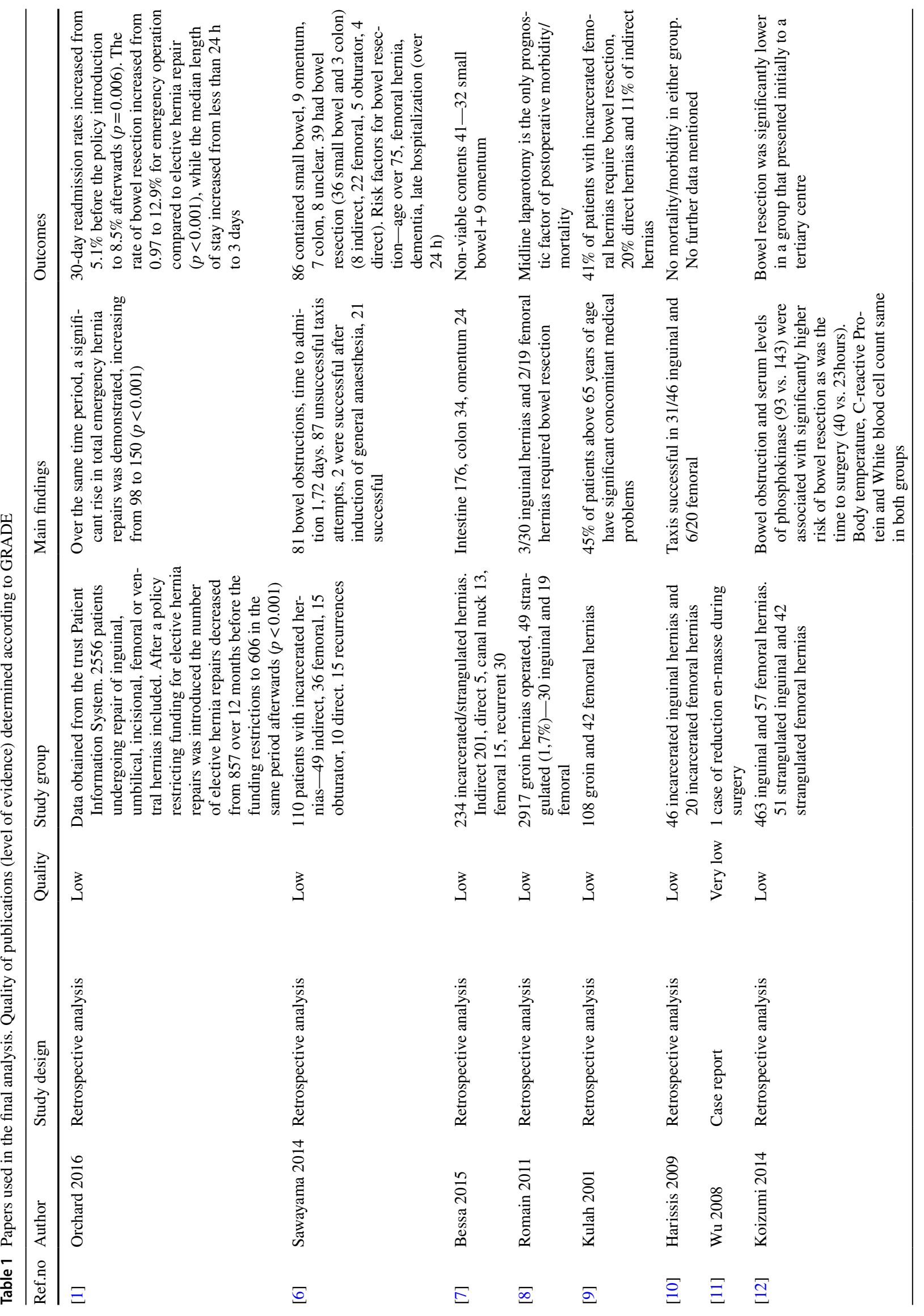


Funding Barbora East is currently receiving a grant from the Czech health research council no. 17-32285A.

\section{Compliance with ethical standards}

Conflict of interest The authors declare no conflict of interest regarding this article.

Ethical approval The authors declare that they did not need any ethical approval.

Human and animal rights This article does not contain any studies with human participants or animals performed by any of the authors.

Informed consent Informed consent as the study is a literature review and did not include any living subjects.

\section{References}

1. Orchard MR, Wright JA, Kelly A et al (2016) The impact of healthcare rationing on elective and emergency hernia repair. Hernia 20(3):405-409

2. HerniaSurge Group (2018) International guidelines for groin hernia management. Hernia 22(1):1-165

3. East B, Kaufmann R, de Beaux AC (2020) A plea for unification of surgical guidelines in the COVID-19 outbreak. Hernia. Apr 30 (Epub ahead of print)

4. The EHS (2020) European hernia society (EHS) guidance for the management of adult patients with a hernia during the COVID-19 pandemic. Hernia. https://doi.org/10.1007/s10029-020-02212-8 (in press)

5. GRADE Working Group (2004) Grading quality of evidence and strength of recommendations. BMJ 328(7454):1490-1494

6. Sawayama H, Kanemitsu K, Okuma T et al (2014) Safety of polypropylene mesh for incarcerated groin and obturator hernias: a retrospective study of 110 patients. Hernia 18(3):399-406

7. Bessa SS, Abdel-fattah MR, Al-Sayes IA et al (2015) Results of prosthetic mesh repair in the emergency management of the acutely incarcerated and/or strangulated groin hernias: a 10-year study. Hernia 19(6):909-914

8. Romain B, Chemaly R, Meyer N et al (2012) Prognostic factors of postoperative morbidity and mortality in strangulated groin hernia. Hernia 16(4):405-410

9. Kulah B, Duzgun AP, Moran M et al (2001) Emergency hernia repairs in elderly patients. Am J Surg 182(5):455-459

10. Harissis HV, Douitsis E, Fatouros M (2009) Incarcerated hernia: to reduce or not to reduce? Hernia 13(3):263-266

11. Wu SC, Wang CC, Sheen-Chen SM (2008) Intestinal obstruction caused by intraoperative mass reduction of an incarcerated inguinal hernia-report of a case. Int J Surg 6(6):e103-e105

12. Koizumi M, Sata N, Kaneda Y et al (2014) Optimal timeline for emergency surgery in patients with strangulated groin hernias. Hernia 18(6):845-848

13. Chen SC, Lee CC, Liu YP et al (2005) Ultrasound may decrease the emergency surgery rate of incarcerated inguinal hernia. Scand J Gastroenterol 40(6):721-724

14. De Simone B, Birindelli A, Ansaloni L et al (2020) Emergency repair of complicated abdominal wall hernias: WSES guidelines. Hernia 24(2):359-368

Publisher's Note Springer Nature remains neutral with regard to jurisdictional claims in published maps and institutional affiliations. 